\title{
Incidence of Venous Thromboembolism Using 64 Channel Multidetector Row Computed Tomography- Indirect Venography and Anti-Coagulation Therapy after Total Knee Arthroplasty in Korea
}

\author{
Kyung-Hyun Park, MD, Sang-Ho Cheon, MD, Ji-Ho Lee, MD and Hee-Soo Kyung, MD \\ Department of Orthopedic Surgery, Kyungpook National University School of Medicine, Daegu, Korea
}

\begin{abstract}
Purpose: This study evaluated the incidence of a venous thromboembolism (VTE) after total knee arthroplasty (TKA) using multidetector row computed tomography-indirect venography (MDCT-indirect venography) and assessed the efficacy of anti-coagulation therapy.

Materials and Methods: We enrolled 118 patients with 126 cases of TKA. The average age of the patients was 68.4 years. We used 64 channel MDCTindirect venography for the detection of VTE. We treated selectively proximal deep vein thrombosis (DVT) or pulmonary thromboembolism (PTE) cases according to the results of MDCT-indirect venography. We re-evaluated the change in VTE using follow-up MDCT-indirect venography after 3 months.

Results: We identified VTE in $35.7 \%$. DVT only was identified in $22.2 \%$ including 8 cases of proximal DVT and 20 cases of distal DVT. PTE without DVT was identified in 4.8\%, and combined DVT and PTE in $8.7 \%$. All patients with PTE were asymptomatic, but 4 DVT patients had signs of leg swelling. After anti-coagulation therapy, 20 patients showed complete resolution in 16 cases, improvement in 3 cases and one case showed a new distal DVT.

Conclusions: The incidence of VTE after primary TKA was 35.7\% in Korea. Furthermore, anti-coagulation therapy for proximal DVT and PTE patients may be a useful method for preventing the occurrence of a fatal PTE.
\end{abstract}

Key words: Total knee arthroplasty, Venous thromboembolism, Deep vein thrombosis, Pulmonary thromboembolism, Multidetector-row computed tomography, Indirect venography.

\section{Introduction}

Deep vein thrombosis (DVT) is a relatively common complication of total hip arthroplasty (THA) and total knee arthroplasty $(\text { TKA })^{1)}$. DVT can lead to pulmonary thromboembolism

Received July 29, 2011; Revised (1st) August 20, 2011;

(2nd) October 4, 2011; Accepted October 17, 2011.

Correspondence to: Hee-Soo Kyung, MD.

Department of Orthopedic Surgery, Kyungpook National University School of Medicine, 130 Dongdeok-ro, Jung-gu, Daegu 700-721, Korea. Tel: +82-53-420-5636, Fax: +82-53-422-6605

Email: hskyung@knu.ac.kr

This is an Open Access article distributed under the terms of the Creative Commons Attribution Non-Commercial License (http://creativecommons.org/licenses/by-nc/3.0/) which permits unrestricted non-commercial use, distribution, and reproduction in any medium, provided the original work is properly cited.
(PTE) and is recognized as one of the most frequent causes of death after arthoplasty ${ }^{2-4)}$. Therefore, the importance of proper diagnosis cannot be overemphasized. Some studies have shown that DVT is less prevalent and PTE is rare in Korean patients after TKA compared to Western countries. However, the incidence of DVT has not been extensively addressed in domestic studies ${ }^{5-8)}$. DVT has been known to occur in $40 \%$ to $70 \%$ of patients without prophylaxis (physical treatment or medication) after major orthopedic surger $y^{9,10}$. On the other hand, Patterson et al. ${ }^{11)}$ reported that complications attributable to the prophylactic anticoagulation therapy (hematomas, wound dehiscence, and intestinal/cerebral hemorrhage) were observed in $45 \%$ of patients after surgery. In addition, thromboprophylaxis requires prolonged hospitalization and the associated complications including cerebral hemorrhage may cause permanent damage. The most common diagnostic modalities for DVT are venography and color Doppler ultrasonography. Unfortunately, venography is invasive and may cause allergic reactions and DVT. 
Color Doppler ultrasonography is not reliable for PTE detection, is less sensitive for lower limb DVT, and has low interobserver reproducibility. Multidetector-row computed tomographyindirect venography (MDCT-indirect venography) is a recent addition to the available methods for the detection of venous thromboembolism (VTE). It provides high quality images, requires a short examination time, and allows for visualization of large areas and reconstruction of three dimensional images. In particular, compared to the conventional 16-channel MDCTindirect venography (10 $\mathrm{mm}$ coverage/rotation), 64-channel MDCT-indirect venography has a higher diagnostic value providing high quality images four times faster (40 $\mathrm{mm}$ coverage/ rotation $)^{7)}$. Therefore, we thought that if MDCT-indirect venography was proven to be a safe, reliable, and time-saving method for early detection of VTE, it would eventually contribute to a reduction in complications resulting from prophylactic anticoagulation therapy.

In this study, we evaluated the incidence of VTE using MDCTindirect venography in patients without prophylaxis after TKA and the results of anticoagulation therapy in patients with proximal DVT or PTE.

\section{Materials and Methods}

All of 118 patients (126 knees) who underwent primary TKA between January 1st 2008 and December 31st 2008 at our institution were enrolled in this study. The exclusion criterion was revision surgery. There were 13 males and 105 females with a mean age of 68.4 years (range, 48 to 83 years) at the time of surgery. The mean body mass index was $23.13 \mathrm{~kg} / \mathrm{m}^{2}$ (range, 16.60 to $33.77 \mathrm{~kg} / \mathrm{m}^{2}$ ). The cause of surgery was degenerative arthritis in 117 cases, rheumatoid arthritis in 7 cases, and osteonecrosis of the medial femoral condyle in 2 cases. In patients with bilateral TKA, there was an interval of $\geq 3$ months between the two operations. If a VTE was identified in these patients, anticoagulation therapy was performed prior to the latter operation. All the patients wore a compression stocking on the unaffected limb and a Jones compression dressing on the affected limb immediately after surgery. On the 2 nd postoperative day, the dressing was replaced by a compression stocking after drainage removal and active ambulation and continuous passive motion exercises were started according to the patient's condition. Prophylactic physical therapy including dorsiflexion and plantar flexion exercises were initiated immediately after surgery in all patients. Prophylactic drug therapy was not used. The presence of symptoms including leg swelling and pain was assessed prior to
MDCT-indirect venography.

MDCT-indirect venography was performed using 64-channel multidetector computed tomography (Aquilion, Toshiba, Nasu Shiobara, Japan) on the 7 th postoperative day ${ }^{3,12,13)}$. A contrast agent was rapidly injected into the brachial vein. When the CT attenuation value was $200-250 \mathrm{HU}$, scanning was performed from the apex of the lung to the costophrenic angle to obtain arterial phase images of the pulmonary artery and the upper abdominal aorta, the early perfusion images. Subsequent scanning was performed from the diaphragm to the foot 140 seconds later to obtain contrast-enhanced venous phase images. Immediately after MDCT-indirect venography, $5 \mathrm{~mm}$ and $0.75 \mathrm{~mm}$ thick axial slices were reconstructed and sent to a radiologist to investigate a filling defect in the vein.

VTE was classified into PTE and DVT and the latter was subcategorized into proximal and distal DVT.

Once a proximal DVT or PTE was diagnosed, warfarin was administered as an anticoagulant. Clexane (Sanofi Aventis, Paris, France, Enoxaparin sodium $1 \mathrm{mg} / \mathrm{kg}$ ) was used at the same time because warfarin is a slow acting drug. A total of $5 \mathrm{mg}$ of warfarin was orally administered and Clexane was subcutaneously injected on two separate occasions on the first day. Thereafter, based on the daily assessment of the international normalized ratio (INR), the use of warfarin was adjusted by $1 \mathrm{mg}$ to maintain the INR in the range of 2 to 3 . It was reduced to $3 \mathrm{mg}$ to reduce hemorrhage if the INR was $\geq 1$.6. If the INR was $\geq 2$ for two consecutive days, Clexane injection was discontinued and warfarin was administered alone. According to ACCP guidelines, the anticoagulant was given until a second MDCT-indirect venography was performed three months later ${ }^{14)}$. If a distal DVT was diagnosed, compression stockings were prescribed without drug therapy and ankle joint exercises were started. On the second MDCT-indirect venography, complete or $\geq 50 \%$ resolution of the lesion was assessed. The mean follow-up period was 12.6 months (range, 7 to 18 months).

\section{Results}

MDCT-indirect venography revealed VTE in 45 (35.7\%) of the cases. DVT only was observed in $22.2 \%$ (proximal DVT in $6.3 \%$ and distal DVT in 15.9\%) (Fig. 1). PTE was observed in $13.5 \%$ of the cases (PTE only in 4.8\% and PTE and DVT in 8.7\%) (Table 1, Fig. 2). Of the 39 cases of DVT, 33 (84.6\%) were found on the operated limb only, 3 (7.7\%) on the contralateral limb only, and 3 cases $(7.7 \%)$ on both limbs. Lower limb swelling was observed in $4(10.2 \%)$ of the DVT cases, in 2 proximal DVT and 2 distal DVT 

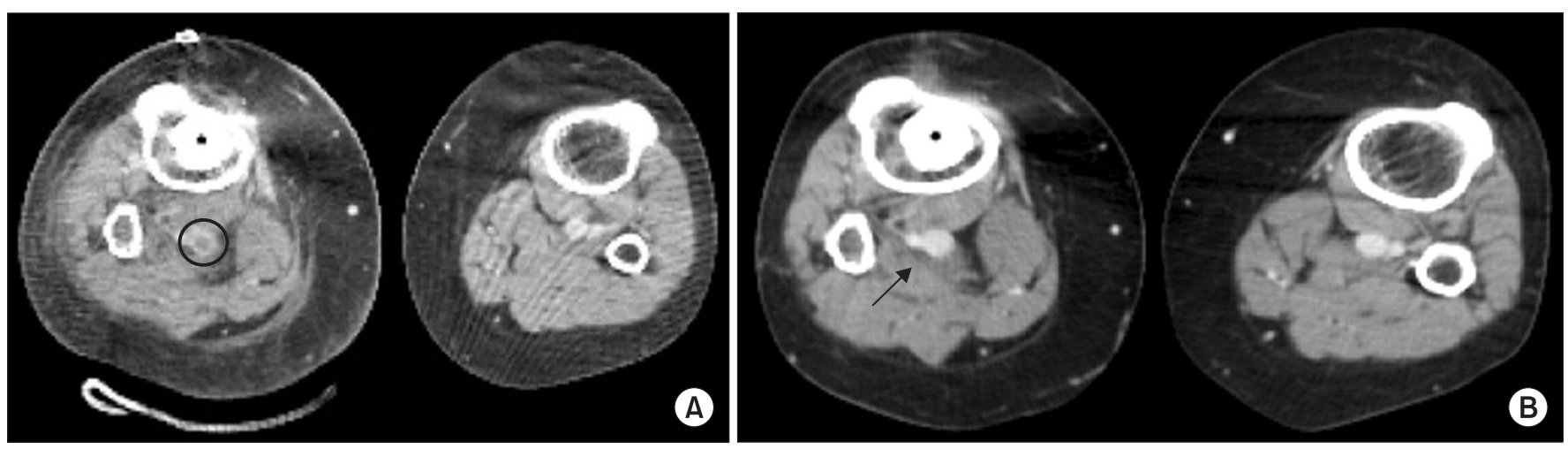

Fig. 1. A 74-year-old woman, who underwent primary total knee arthroplasty, was diagnosed as having deep vein thrombosis in the right popliteal vein at postoperative day 7. (A) Deep vein thrombosis in the right popliteal vein was found by multidetector row computed tomography (MDCT)venography (circle). (B) Follow-up MDCT-venography at 3 months postoperatively showed complete resolution of deep vein thrombosis of the right popliteal vein after anti-coagulation therapy (arrow).

Table 1. The Prevalence of VTE Diagnosed on Day 7 after Total Knee Arthroplasty

\begin{tabular}{|c|c|c|c|c|}
\hline & & & Cases & $\begin{array}{c}\text { Incidence }(\%) \\
(n=126)\end{array}$ \\
\hline No VTE & & & 81 & 64.30 \\
\hline \multirow[t]{8}{*}{ VTE } & & & 45 & 35.70 \\
\hline & DVT only & Proximal DVT & 8 & 6.30 \\
\hline & & Distal DVT & 20 & 15.90 \\
\hline & PTE only & & 6 & 4.80 \\
\hline & DVT and PTE & & 11 & 8.70 \\
\hline & & Proximal DVT & 1 & 0.79 \\
\hline & & Distal DVT & 8 & 6.35 \\
\hline & & $\begin{array}{l}\text { Proximal \& distal } \\
\text { DVT }\end{array}$ & 2 & 1.58 \\
\hline Total & & & 126 & 100.00 \\
\hline
\end{tabular}

VTE: venous thromboembolism, DVT: deep vein thrombosis, PTE: pulmonary thromboembolism.

cases. PTE was asymptomatic in all cases.

Anticoagulation therapy was performed in 25 cases, in 17 PTE and 8 proximal $\mathrm{DVT}^{15)}$. Of these, 20 cases were available for a second MDCT-indirect venography, which showed complete resolution in 16 cases (80\%), $\geq 50 \%$ resolution in 3 cases (15\%), and PTE resolution but occurrence of distal DVT in 1 case (5\%).

Surgical site swelling was observed in 4 cases and headache occurred in 1 case during anticoagulation therapy, which improved without particular complications in five days. Severe intramuscular hematoma was found in 1 case, which improved in ten days after discontinuation of the anticoagulation therapy and initiation of conservative treatment. Neither a fatal PTE nor other complications were observed, but the mean hospitalization period became longer by 7 days (range, 5 to 10 days) for INR control.

\section{Discussion}

VTE is a common complication after orthopedic surgery that presents with leg swelling and pain. It is a potentially serious disease that can lead to fatal PTE. This is why early diagnosis of VTE is crucial. Venography and color Doppler ultrasonography have been commonly used for early detection of DVT. However, their efficacy has been questioned due to the inherent disadvantages of these techniques ${ }^{16,17)}$. Venography is highly effective for DVT detection, but it is invasive, associated with allergic reactions and DVT occurrence, and unhelpful for PTE detection. Color Doppler ultrasonography requires changes in the patient's positions (supine and prone positions) during examination and is not useful for the assessment of inferior vena cava, iliac vein, and pulmonary vein. In contrast, MDCTvenography can be performed within a short examination time without positional changes and can visualize not only the lower limb vessels but also the thoracic, abdominal, and pelvic vessels ${ }^{6}$. The sensitivity and specificity of dual-detector CT venography for diagnosing DVT were $93 \%$ and $97 \%$, respectively, in a study by Coche et al. ${ }^{18)}$ and $89 \%$ and $94 \%$, respectively, in a study by Duwe et al. ${ }^{19)}$ The sensitivity and specificity of multi-slice spiral CT was $94.3 \%$ and $92.1 \%$, respectively, in a study by Muller et $\mathrm{al}^{20)}$ The advantage of 64-channel MDCT-indirect venography is that it provides high quality images four times faster than the conventional 16-channel MDCT-venography. Therefore, we used 64-channel MDCT-indirect venography for the diagnosis of VTE 

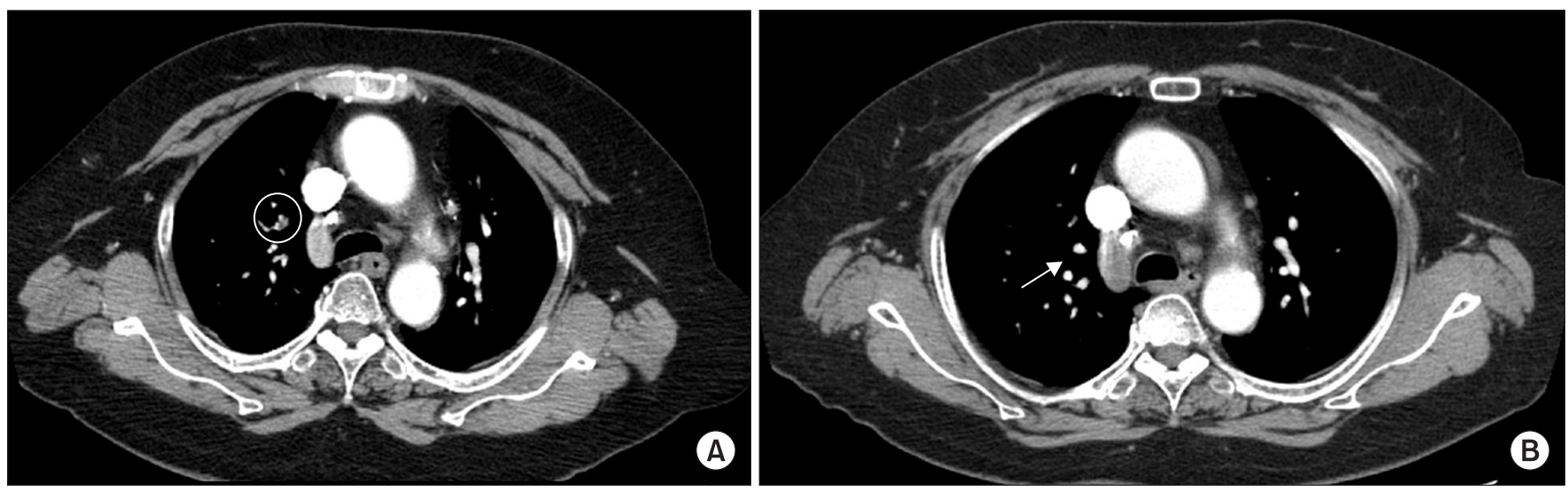

Fig. 2. A 66-year-old woman, who underwent primary total knee arthroplasty, was diagnosed as having a pulmonary thromboembolism in the right upper lobe, at 7 days postoperatively. (A) Thromboembolism was detected by multidetector row computed tomography (MDCT)-venography in the right upper lobar segmental pulmonary artery (circle). (B) Follow-up MDCT-venography at 3 months postoperatively showed complete resolution of pulmonary thromboembolism in the right upper lobar segmental pulmonary artery after anti-coagulation therapy (arrow).

in this study.

Internationally, the prevalence of DVT is $50-70 \%, 10-18 \%$ of which leads to PTE that has a mortality rate of $0.2-0.7 \%{ }^{21}$. In a domestic study, Song et al. ${ }^{22)}$ reported that DVT was diagnosed in $11 \%$ of the TKA patients with use of venography. In the studies by Kim et al. ${ }^{6}$ and Song et al. ${ }^{8)}$, 64-channel MDCTdirect venography revealed a DVT in $34 \%$ of 140 patients. Of the DVT patients, $10.4 \%(5 / 48)$ had symptoms and $30 \%$ had proximal DVT. Although PTE was not found in any of the patients, asymptomatic PTEs might not have been identified due to the use of direct venography. Lee et al." used 16-channel MDCT-indirect venography to diagnose DVT in 63 patients (71 cases) with THA or TKA (32 THA cases and 39 TKA cases). In their study, DVT was diagnosed in $46.5 \%, 25.7 \%$ of which was symptomatic. In this study, VTE was identified in $35.7 \%, 10.2 \%$ of which was symptomatic. The incidence of asymptomatic PTE was $13.5 \%$, which was relatively higher than that in other domestic studies. In our opinion, this does not indicate that there has been an increase in the occurrence of PTE. We think that this is attributable to the development of the diagnostic tool: direct venography is not useful for PTE detection and 64-channel MDCT-indirect venography is a more advanced technique compared to the 16-channel MDCT-indirect venography.

Recently, prophylactic use of anticoagulation for DVT and PTE prevention has been recommended in the West ${ }^{23)}$. Horii et al. ${ }^{15)}$ suggested the use of anticoagulants for proximal DVT and PTE because PTEs were less correlated with distal DVTs in the study. However, prophylactic anticoagulation therapy does not necessarily result in good clinical results. DVT can be observed in $10-20 \%$ of the arthroplasty patients on prophylactic anticoagulation therapy and PTE in $1 \%^{24,25)}$. According to Warwick and Whitehouse ${ }^{26)}$, chemical prophylaxis reduces the veonographic prevalence of DVT, but it is uncertain whether this affects the incidence of symptoms. Patterson et al. ${ }^{11)}$ reported that $45 \%$ of patients on anticoagulation therapy had complications including hematomas, surgical site dehiscence, and intestinal/cerebral bleeding. In addition, cerebral bleeding and prolonged hospitalization may result in permanent disabilities. The incidence of fatal PTE in patients without prophylactic anticoagulation was $1(0.2 \%)$ in 499 patients according to Khaw et al. ${ }^{27}, 0(0 \%)$ in 244 patients according to $\mathrm{Kim}^{28}$, and $1(0.1 \%)$ in 1,000 patients according to Warwick and Whitehouse ${ }^{26)}$. On the other hand, Song et al. ${ }^{8)}$ reported that 12 TKA patients with symptomatic DVT that was diagnosed using MDCT-venography obtained improvement with anticoagulant therapy and no particular complications were observed. In this study, PTE was observed in 17 cases (13.5\%), none of which presented with fatal symptoms. In addition, anticoagulation led to improvement in 95\% of cases with proximal DVT or PTE.

MDCT-indirect venography can be used to determine the necessity of anticoagulation therapy for VTE after TKA considering that the frequency of VTE and PTE after TKA is low in the Asian population ${ }^{28)}$ and thromboprophylaxis measures using anticoagulants can increase the risk of bleeding. However, MDCT-indirect venography is costly and may cause contrast agent-induced renal failure. Therefore, we believe that MDCT-indirect venography should be performed in patients with high risk of $\mathrm{VTE}^{29,30)}$ and anticoagulation therapy should be selectively administered based on the assessment results to prevent fatal PTE. The implication of our study is that we 
assessed the incidence of VTE in the total study population with use of MDCT-indirect venography that is effective for rapidly diagnosing both DVT and PTE at the same time.

Our study had limitations. There were no comparisons with other studies and the number of patients for follow-up venography was small. Considering that the use of MDCTindirect venography can be restricted in some patients due to its disadvantages, more follow-up studies including a larger study population and comparisons should be performed.

\section{Conclusions}

MDCT-indirect venography revealed that the incidence of VTE after TKA was $35.7 \%$ in our patients. We think that anticoagulation therapy for proximal DVT and PTE can be a useful method for preventing fatal PTE.

\section{References}

1. Ko PS, Chan WF, Siu TH, Khoo J, Wu WC, Lam JJ. Deep venous thrombosis after total hip or knee arthroplasty in a "low-risk" Chinese population. J Arthroplasty. 2003;18:1749.

2. Mont MA, Jones LC, Rajadhyaksha AD, Shuler MS, Hungerford DS, Sieve-Smith L, Wang P, Cordista AG, Glueck CJ. Risk factors for pulmonary emboli after total hip or knee arthroplasty. Clin Orthop Relat Res. 2004;(422):15463.

3. White RH, Henderson MC. Risk factors for venous thromboembolism after total hip and knee replacement surgery. Curr Opin Pulm Med. 2002;8:365-71.

4. Yoo MC, Cho YJ, Kim KI, Im YJ, Cho KY, Ryu KN. Incidence of deep vein thrombosis after major hipsurgery and diagnostic value of D-dimer. J Korean Orthop Assoc. 2006;41:103-9.

5. Kim YH, Kim JS. Incidence and natural history of deepvein thrombosis after total knee arthroplasty. A prospective, randomised study. J Bone Joint Surg Br. 2002;84:566-70.

6. Kim YJ, Hur CI, Song EK, Seon JK, Park SJ, Cho SB, Cho YJ. Availability of D-dimer test for the diagnosis of deep vein thrombosis after total knee arthroplasty. J Korean Orthop Assoc 2007;42:523-9.

7. Lee Yg, Kim Mk, Cho KJ, Kim Hg, Cho JM, Jeon Ys, Kim Jh. The diagnostic availability of multidetector-row computed tomography (MDCT) in deep vein thrombosis developed after joint arthroplasty. J Korean Orthop Assoc. 2006;41:134-9.
8. Song EK, Seon JK, Park SJ, Cho SB, Choi MS. Diagnosis of the deep vein thrombosis with multidetector-row computed tomographic venography after total knee arthroplasty. J Korean Orthop Assoc 2008;43:294-300.

9. Stringer MD, Steadman CA, Hedges AR, Thomas EM, Morley TR, Kakkar VV. Deep vein thrombosis after elective knee surgery. An incidence study in 312 patients. J Bone Joint Surg Br. 1989;71:492-7.

10. Turpie AG, Levine MN, Hirsh J, Carter CJ, Jay RM, Powers PJ, Andrew M, Hull RD, Gent M. A randomized controlled trial of a low-molecular-weight heparin (enoxaparin) to prevent deep-vein thrombosis in patients undergoing elective hip surgery. N Engl J Med. 1986;315:925-9.

11. Patterson BM, Marchand R, Ranawat C. Complications of heparin therapy after total joint arthroplasty. J Bone Joint Surg Am. 1989;71:1130-4.

12. Leizorovicz A; SMART Venography Study Steering Committee. Epidemiology of post-operative venous thromboembolism in Asian patients. Results of the SMART venography study. Haematologica. 2007;92:1194-200.

13. Maynard MJ, Sculco TP, Ghelman B. Progression and regression of deep vein thrombosis after total knee arthroplasty. Clin Orthop Relat Res. 1991;(273):125-30.

14. Kearon C, Kahn SR, Agnelli G, Goldhaber S, Raskob GE, Comerota AJ; American College of Chest Physicians. Antithrombotic therapy for venous thromboembolic disease: American College of Chest Physicians Evidence-Based Clinical Practice Guidelines (8th Edition). Chest. 2008;133(6 suppl):454S-545S.

15. Horii Y, Yoshimura N, Hori Y, Takaki S, Takano T, Inagawa $\mathrm{S}$, Aoyama H. Correlation between the site of pulmonary embolism and the extent of deep vein thrombosis: evaluation by computed tomography pulmonary angiography and computed tomography venography. Jpn J Radiol. 2011;29:171-6.

16. Borris LC, Christiansen HM, Lassen MR, Olsen AD, Schott P. Comparison of real-time B-mode ultrasonography and bilateral ascending phlebography for detection of postoperative deep vein thrombosis following elective hip surgery. The Venous Thrombosis Group. Thromb Haemost. 1989;61:363-5.

17. Cronan JJ, Dorfman GS, Scola FH, Schepps B, Alexander J. Deep venous thrombosis: US assessment using vein compression. Radiology. 1987;162:191-4.

18. Coche EE, Hamoir XL, Hammer FD, Hainaut P, Goffette PP. Using dual-detector helical CT angiography to detect deep 
venous thrombosis in patients with suspicion of pulmonary embolism: diagnostic value and additional findings. AJR Am J Roentgenol. 2001;176:1035-9.

19. Duwe KM, Shiau M, Budorick NE, Austin JH, Berkmen YM. Evaluation of the lower extremity veins in patients with suspected pulmonary embolism: a retrospective comparison of helical CT venography and sonography. 2000 ARRS Executive Council Award I. American Roentgen Ray Society. AJR Am J Roentgenol. 2000;175:1525-31.

20. Muller C, Kopka L, Funke M, Funke C, Grabbe E. Diagnosis of lung embolism and underlying venous thrombosis in multi-slice spiral CT. Rofo. 2001;173:528-35.

21. Jimenez MA, Trousdale RT. Thromboembolic disease in total knee arthroplasty. Instr Course Lect. 2001;50:415-9.

22. Song EK, Kim JK, Lee KB, Seon JK. Deep vein thrombosis after total knee replacement: icidence and correlation with clinical risk factors. J Korean Knee Soc. 1998;10:18-22.

23. Weijl NI, Rutten MF, Zwinderman AH, Keizer HJ, Nooy MA, Rosendaal FR, Cleton FJ, Osanto S. Thromboembolic events during chemotherapy for germ cell cancer: a cohort study and review of the literature. J Clin Oncol. 2000;18:2169-78.

24. Leyvraz PF, Richard J, Bachmann F, Van Melle G, Treyvaud JM, Livio JJ, Candardjis G. Adjusted versus fixed-dose subcutaneous heparin in the prevention of deep-vein thrombosis after total hip replacement. N Engl J Med. 1983;309:954-8.

25. Sharrock NE, Brien WW, Salvati EA, Mineo R, Garvin K, Sculco TP. The effect of intravenous fixed-dose heparin during total hip arthroplasty on the incidence of deep-vein thrombosis. A randomized, double-blind trial in patients operated on with epidural anesthesia and controlled hypotension. J Bone Joint Surg Am. 1990;72:1456-61.

26. Warwick DJ, Whitehouse S. Symptomatic venous thromboembolism after total knee replacement. J Bone Joint Surg Br. 1997;79:780-6.

27. Khaw FM, Moran CG, Pinder IM, Smith SR. The incidence of fatal pulmonary embolism after knee replacement with no prophylactic anticoagulation. J Bone Joint Surg Br. 1993;75:940-1.

28. Kim YH. The incidence of deep vein thrombosis after cementless and cemented knee replacement. J Bone Joint Surg Br. 1990;72:779-83.

29. Haas SB, Barrack RL, Westrich G, Lachiewicz PF. Venous thromboembolic disease after total hip and knee arthroplasty. J Bone Joint Surg Am. 2008;90:2764-80.

30. Carter K. NICE guidance to reduce the risk of venous thromboembolism in hospital patients. Nurs Times. 2010;106:15. 